\title{
Product of $A_{p}$ Weight Functions
}

\author{
Vinod Parajuli, Santosh Ghimire \\ Department of Engineering Science and Humanities, Pulchowk Campus, Institute of Engineering \\ Tribhuvan University, Nepal \\ Corresponding author: santoshghimire@ioe.edu.np
}

\begin{abstract}
In this paper, we first define $A_{p}$ weight functions and then show that finite product of weight functions each raised with some power whose sum is one is also an $A_{p}$ weight function.
\end{abstract}

Keywords: weight function, Holder's inequality, Maximal function.

\section{Introduction}

In 1970, Muckenhoupt characterized positive functions $w$ for which the Hardy-Littlewood maximal operator $M$ maps $L^{\mathrm{p}}\left(\mathrm{R}^{\mathrm{n}}, \mathrm{w}(\mathrm{x}) \mathrm{dx}\right)$ to itself. Muckenhoupt's characterization actually gave the better understanding of theory of weighted inequalities which then led to the introduction of $\mathrm{A}_{\mathrm{p}}$ class and consequently the development of weighted inequalities. Weighted inequalities are used widely in harmonic analysis. For more about the theory of weights and applications in harmonic analysis, refer $[1,4]$.

In order to prove the result, some definitions and results are in order:

Definition: A locally integrable function on $\mathrm{R}^{\mathrm{n}}$ that takes values in the interval $(0, \infty)$ almost everywhere is called a weight. So by definition a weight function can be zero or infinity only on a set whose Lebesgue measure is zero.

We use the notation $w(E)=\int_{E} w(x) d x$ to denote the w-measure of the set $\mathrm{E}$ and we reserve the notation $\mathrm{L}^{\mathrm{p}}\left(\mathrm{R}^{\mathrm{n}}, \mathrm{w}\right)$ or $\mathrm{L}^{\mathrm{p}}(\mathrm{w})$ for the weighted $\mathrm{L}^{\mathrm{p}}$ spaces. We note that $\mathrm{w}(\mathrm{E})<\infty$ for all sets $\mathrm{E}$ contained in some ball since the weights are locally integrable functions.

Definition: A function $w(x) \geq 0$ is called an $A_{1}$ weight if there is a constant $C_{1}>0$ such that

$$
\mathrm{M}(\mathrm{w})(\mathrm{x}) \leq \mathrm{C}_{1} \mathrm{w}(\mathrm{x})
$$

where $\mathrm{M}(\mathrm{w})$ is uncentered Hardy-Littlewood Maximal function given by

$$
M(w)(x)=\sup _{x \in B} \frac{1}{|B|} \int_{B} w(t) d t .
$$

If $\mathrm{w}$ is an $\mathrm{A}_{1}$ weight, then the quantity (which is finite) given by 


$$
[w]_{A_{1}}=\sup _{Q \text { cubesinR }}\left(\frac{1}{|Q|} \int_{Q}|w(t)| d t\right)\left\|w^{-1}\right\|_{L^{\infty}(Q)}
$$

is called the $A_{1}$ characteristic constant of $\mathrm{w}$.

Definition: Let $1<\mathrm{p}<\infty$. A weight $\mathrm{w}$ is said to be of class $\mathrm{A}_{\mathrm{p}}$ if $[w]_{A_{p}}$ is finite where $[w]_{A_{p}}$ is defined as

$$
[w]_{A_{p}}=\sup _{Q \text { cubesin } R^{n}}\left(\frac{1}{|Q|} \int_{Q}|w(x)| d x\right)\left(\frac{1}{|Q|} \int_{Q}|w(x)|^{\frac{-1}{p-1}} d x\right)^{p-1}
$$

We remark that in the above definition of $A_{1}$ and $A_{p}$ one can also use set of all balls in $R^{n}$ instead of all cubes in $R^{n}$. Readers are suggested to read [4] for motivation, properties of $A_{p}$ weights and much more about the $A_{p}$ weights. Also refer [2] and [3] for more properties on $A_{1}$ and $A_{p}$ weight function.

\section{Holder's inequality}

Let $\mathrm{p}$ and $\mathrm{q}$ be two real numbers such that $\mathrm{p}>1$ and $\frac{1}{p}+\frac{1}{q}=1$ and if $f \in L^{p}$ and $g \in L^{q}$. Then $f . g \in L^{1}$ and

$\int f g d x \leq\left(\int|f|^{p} d x\right)^{\frac{1}{p}}\left(\int|g|^{q} d x\right)^{\frac{1}{q}}$

Now we state our main result.

Suppose that weight $w_{j} \in A_{P_{j}}$ with $1 \leq j \leq m$ for some $1 \leq p_{1} \ldots . p_{m}<\infty$ and let $0<$ $\theta_{1} \ldots . \theta_{m}<1$ be such that $\sum_{j=1}^{m} \theta_{j}=1$. We then show that the product function given by

$$
W:=\prod_{j=1}^{m} w_{j}^{\theta_{j}}
$$

is an $\mathrm{A}_{\mathrm{p}}$ weight function where $\mathrm{p}$ is the maximum value of $p_{1}, \ldots \ldots, p_{m}$. The proof will be done in following steps:

(i) We prove that $w_{j} \in A_{P}$ for all $\mathrm{j}$.

(ii) We show that the following inequality holds:

$$
\frac{1}{|Q|} \int_{Q} \prod_{j=1}^{m} w_{j}^{\theta_{j}}(x) d x \leq \prod_{j=1}^{m}\left(\frac{1}{|Q|} \int_{Q} w_{j}(x) d x\right)^{\theta_{j}}
$$

(iii) We will use (ii) and the Holder's inequality to show 
118 Product of Ap Weight Functions

$$
[W]_{A_{p}} \leq \prod_{j=1}^{m}\left(\left[w_{j}\right]_{A_{p}}\right)^{\theta_{j}}
$$

(iv) Finally by (i) and (iii) we prove that $W \in A_{p}$.

Since $p_{j} \leq P$ for all $\mathrm{j}$, using the decreasing nature of $w_{j}$, we have $\left[w_{j}\right]_{A_{p}} \leq\left[w_{j}\right]_{A_{p_{j}}}$ for all $\mathrm{j}$. This proves (i). To prove (ii) we do as follows. If $w_{j}=0$ for some $\mathrm{j}$ then the equality holds. Assuming $w_{j} \neq 0$ for all $\mathrm{j}$ and letting $x_{j}=\frac{w_{j}(x)}{\frac{1}{|Q|} \int_{Q} w_{j}(x) d x}$ one gets

$$
\log \left(\prod_{j=1}^{m} x_{j} \theta_{j}\right)=\sum_{j=1}^{m} \theta_{j} \log \left(x_{j}\right) \leq \log \left(\sum_{j=1}^{m} \theta_{j} x_{j}\right)
$$

We note that we used the concavity of $\log (x)$ in the above expression. Since $\log (x)$ is an increasing function, it follows that

$\prod_{j=1}^{m} x_{j} \theta_{j} \leq \sum_{j=1}^{m} \theta_{j} x_{j}$.

This implies

$$
\frac{1}{|Q|} \int_{Q} \prod_{j=1}^{m} x_{j}^{\theta_{j}} d x \leq \frac{1}{|Q|} \int_{Q} \sum_{j=1}^{m} \theta_{j} x_{j} d x=\sum_{j=1}^{m} \theta_{j} \frac{1}{|Q|} \int_{Q} x_{j} d x=\sum_{j=1}^{m} \theta_{j}=1 .
$$

From the above inequality (ii) follows. Finally we prove (iii).

Let $G:=\left(\frac{1}{|Q|} \int_{Q} W d x\right)\left(\frac{1}{|Q|} \int_{Q} W^{\frac{-1}{p-1}} d x\right)^{p-1}$. By (ii) we have,

$$
G \leq\left[\prod_{j=1}^{m}\left(\frac{1}{|Q|} \int_{Q}\left|w_{j}(x)\right| d x\right)^{\theta_{j}}\right]\left(\frac{1}{|Q|} \int_{Q} W^{\frac{-1}{p-1}} d x\right)^{p-1}
$$

We write

$W^{\frac{-1}{p-1}}=\prod_{j=1}^{m}\left(\frac{1}{|Q|} \int_{Q} w_{j}^{\frac{-1}{p-1}} d x\right)^{\theta_{j}}$

Let $s=\frac{1}{\theta_{1}}$ and $\frac{1}{s}+\frac{1}{s^{\prime}}=1$. Applying the Holder's inequality, we obtain

$$
\begin{aligned}
\int_{Q} W^{\frac{-1}{p-1}} d x & \leq\left(\int_{Q} w_{1}^{\frac{-1}{p-1}}(x) d x\right)^{\frac{1}{s}}\left[\int_{Q} \prod_{j=2}^{m}\left(w_{j}^{\frac{-1}{p-1}}(x)\right)^{\theta_{j} s^{\prime}} d x\right]^{\frac{1}{s^{\prime}}} \\
& =\left(\int_{Q} w_{1}^{\frac{-1}{p-1}}(x) d x\right)^{\theta_{1}}\left[\int_{Q} \prod_{j=2}^{m}\left(w_{j}^{\frac{-1}{p-1}}(x)\right)^{\frac{\theta_{j}}{1-\theta_{1}}} d x\right]^{1-\theta_{1}}
\end{aligned}
$$


$\int_{Q} W^{\frac{-1}{p-1}} d x \leq \prod_{j=1}^{2}\left(\frac{1}{|Q|} \int_{Q} w_{j}^{\frac{-1}{p-1}} d x\right)^{\theta_{j}}\left[\int_{Q} \prod_{j=3}^{m}\left(w_{j}^{\frac{-1}{p-1}}(x)\right)^{\frac{\theta_{j}}{1-\sum_{j=1}^{2} \theta_{j}}} d x\right]^{1-\sum_{j=1}^{2} \theta_{j}}$

Continuing in this manner, one get

$$
\int_{Q} \prod_{j=1}^{m}\left(w_{j}^{\frac{-1}{p-1}}\right)^{\theta_{j}} d x \leq C \prod_{j=1}^{m}\left(\int_{Q} w_{j}^{\frac{-1}{p-1}} d x\right)^{\theta_{j}}
$$

where $\mathrm{C}$ is a constant. Therefore, by (i),

$$
G \leq C \prod_{j=1}^{m}\left[\left(\frac{1}{|Q|} \int_{Q} w_{j}(x) d x\right)\left(\frac{1}{|Q|} \int_{Q} w_{j}^{\frac{-1}{p-1}} d x\right)^{P-1}\right]^{\theta_{j}}
$$

Taking supremum over the cube $\mathrm{Q}$ in the above inequality, we get $W \in A_{p}$.

\section{References}

[1] Ba nelos R and Moore CN (1991), Probabilistic Behavior of Harmonic Functions, Birkhauser Verlag.

[2] Ghimire S (2014), Weighted Inequality, Journal of Institute of Engineering, 10(1), 121-124.

[3] Ghimire S (2014), Two Different Ways to Show a Function is an $\mathrm{A}_{1}$ Weight Function, The Nepali Mathematical Sciences Report, 33(1 \& 2), 2014.

[4] Grafakos L (2009), Modern Fourier Analysis, Second Edition, Springer. 